\title{
Sustainable Intercropping Association of Little Millet (Panicum sumatrense L.) in Niger (Guizotia abyssinica Cass)
}

\author{
R. D. Nigade*, R. L. Bhilare and S. V. Bagade
}

Mahatma Phule Krishi Vidyapeeth, College of Agriculture, Pune, Maharashtra 05, India

*Corresponding author

\section{A B S T R A C T}

\begin{tabular}{|l|}
\hline Ke y w o r d s \\
$\begin{array}{l}\text { Intercropping } \\
\text { systems, little millet } \\
\text { grain equivalent } \\
\text { yield, LER. }\end{array}$ \\
\hline Article Info \\
$\begin{array}{l}\text { Accepted: } \\
\text { 15 May 2020 } \\
\text { Available Online: } \\
\text { 10 June } 2020\end{array}$ \\
\hline
\end{tabular}

A field experiment was conducted at the Zonal Agricultural Research Station, Shenda Park, Kolhapur (Maharashtra) during kharif 2016-17 to 2018-9 to study the performance of intercropping of niger oilseed in little millet. The experiment was laid out in randomized completely block design with nine treatment combinations replicated thrice. The three year pooled data revealed that growing of little millet + niger in 4:1 row proportion recorded highest gross (Rs.45156/-) and net monetary returns (Rs.12298/-) with maximum benefit cost ratio (1.37) and little millet grain equivalent yield (10.93 q ha ${ }^{-1}$ ) with maximum LER value (1.38) compared to other intercropping treatments. The increased net monetary returns and grain equivalent yield were 54.98 and 4.10 per cent over sole crop of little millet, respectively.

\section{Introduction}

Little millet (Panicum sumatrense $\mathrm{L}$ ) and Niger (Guizotia abyssinica cass) are important kharif crops on shallow entisols of Sub-montane zone and Ghat zone of Maharashtra. Little millet is quick growing and early maturing crop. Growing of niger crop around the boundaries of farm and as mix crop in little millet crop is common practice particularly in Konkan and Nasik region of Maharashtra. Intercropping of different cereals, millets, pulses and oilseed crops simultaneously on the same piece of land with or with without any row proportion will minimizes the risk of crop failures, acts as barrier for pests, improves soil fertility and makes the farmer self-sufficient (Manjunath and Salakinkop, 2017).

In intercropping system, the competitive effects between main and intercrop depends on the rooting pattern, canopy structure and days to maturity. The basic idea of intercropping is not only that two or more crop species grown together can exploit the 
resources better than either of them grown separately, but also that the inherent risk in agriculture and more so under rainfed conditions are buffered to some extent called as biological insurance. Mixed or intercropping is common as a means of insurance and risk minimization.

In particular, information on little millet intercropping is not available from Maharashtra, despite the crop is grown at recommended spacing $(30 \mathrm{~cm})$ in consideration to all crops taken as intercrop. With these considerations in view, the experiment was conducted to assess the feasibility of raising niger as intercrop with little millet at different row ratios under rainfed conditions on shallow entisols of Sub montane and Ghat zone of Maharashtra.

\section{Materials and Methods}

The investigation was conducted during kharif seasons for three years 2016-17 to 2018-19 at the Zonal Agricultural Research Station, Shenda Park, Kolhapur (Maharashtra) at latitude of $16^{0} 43^{\prime} \mathrm{N}$, longitude $74^{0} 14^{\prime} \mathrm{E}$ and altitude $574 \mathrm{~m}$ above mean sea level. It comes under Sub-montane Zone of Maharashtra. The experimental site was silty loam with $\mathrm{pH}-7.20$, E.C- $0.15 \mathrm{dSm}^{-1}$, organic carbon 0.48 per cent. The available nutrient status was low in $\mathrm{N}\left(210 . \mathrm{kg} \mathrm{ha}^{-1}\right)$, medium in $\mathrm{P}\left(12.3 \mathrm{~kg} \mathrm{ha}^{-1}\right)$ and low in $\mathrm{K}\left(155 \mathrm{~kg} \mathrm{ha}^{-1}\right)$. The total annual rainfall received during 2016, 2017 and 2018 were $1067.6 \mathrm{~mm}$ in 59 rainy days, $972.2 \mathrm{~mm}$ in 70 rainy days and $1252.0 \mathrm{~mm}$ in 66 rainy days respectively. The dry spell during grand growth phase (33 to 35 MW) affects on yield of crop in all the years. The present experiment was laid out in randomized completely block design with nine treatment combinations replicated thrice. The treatments included in the experiment were $T_{1}$ - Sole little millet, $T_{2}$ - Sole niger, $T_{3}-$ Little millet + niger $2: 1, \mathrm{~T}_{4}$ - Little millet + niger $4: 1, \mathrm{~T}_{5^{-}}$Little millet + niger $6: 1, \mathrm{~T}_{6^{-}}$ Little millet + niger $2: 2, \mathrm{~T}_{7}$ - Little millet + niger $4: 2, \mathrm{~T}_{8^{-}}$Little millet + niger $6: 2$ and $\mathrm{T}_{9^{-}}$ Farmers practice. The gross plot size was 4.00 $\mathrm{x} 3.0 \mathrm{~m}^{2}$ and net plot of size was $4.20 \mathrm{~m} \mathrm{X}$ $2.40 \mathrm{~m}$. The seed rate of $3 \mathrm{~kg} \mathrm{ha}^{-1}$ was used for sowing at spacing of $30 \mathrm{~cm} \mathrm{x} 7.5 \mathrm{~cm}$ in all treatments. The variety used were Phule Ekadahi and Phule Karala for little millet and niger, respectively. On the same day both the crops were sown. The crop was fertilized with

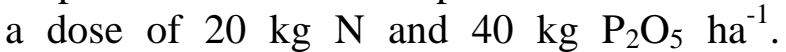
Nitrogen supplied as urea, phosphorus as SSP. The tabulated data were statistically processed by standard method of analysis of variance for the randomized block design and test of significance as given by Panse and Sukhatme (1985). It was further computed in terms of little millet equivalent yield and land equivalent ratio (LER) as described by Willey (1979). The intercropping system was also evaluated on the basis of different economical parameters viz., gross returns (Rs. ha-1), net returns (Rs. ha-1) and B:C ratio.

\section{Results and Discussion}

\section{Growth parameters}

The growth parameters of little millet observed in sole and intercropping treatments were differed significantly (Table 1). Growing of little millet as an sole crop with normal row spacing $(30 \mathrm{~cm})$ recorded significantly highest plant height $(94.9 \mathrm{~cm})$, number of tillers (8.8) and length panicle (28.6). Tripathi and Kushwaha (2013) reported that yield were substantially higher in sole crop than that of intercrop.

\section{Grain and straw yield}

The data presented in Table 2 reveals that sole crop of little millet recorded the higher grain and straw yield (9.78 and $13.16 \mathrm{q} \mathrm{ha}^{-1}$ ), which showed significant superiority over all other 
treatments. Amongst intercropping system, significantly more rain yield of little millet yield $\left(7.78 \mathrm{q} \mathrm{ha}^{-1}\right)$ was recorded with the treatment $\mathrm{T}_{4}$ i.e. little + niger (4:1) than all the treatments except treatments $\mathrm{T}_{6}$, where it was found at par. As regards to straw yield, significantly higher straw yield of $9.65 \mathrm{q} \mathrm{ha}^{-1}$ was obtained with the treatment $\mathrm{T}_{6}$ i.e. little + niger (6:1) as compared to other intercropping systems, however it was at par with $\mathrm{T}_{7}, \mathrm{~T}_{8}$ and $\mathrm{T}_{3}$. The highest value of intercrop yield was observed with the treatment little millet intercropping in 4:1 proportions with niger $\left(1.51 \mathrm{q} \mathrm{ha}^{-1}\right)$. Similar findings were reported by Shashidhar et al., (2000), Ugale et al., (2009) and Patil et al., (2010), Sharmili and Manoharan (2018).

\section{Grain equivalent yield and LER}

The significantly higher little millet equivalent yield of $10.93 \mathrm{q} \mathrm{ha}^{-1}$ was recorded with intercropping of little millet + niger in 4:1 than all other intercropping treatments except treatment $T_{7}$, where yield found at par with each other. The same treatment $\left(\mathrm{T}_{4}\right)$ also observed maximum value of land equivalent ratio (LER) of 1.38 (Table 3) implying that $38 \%$ more land would be required as sole crops to produce the yield obtained under intercrop situations. The obvious reason for yield advantage in intercropping system was due to the fact that the component crops differed in utilization of growth resources and converting them more efficiently resulting in higher yield per unit area than that produced by the sole crops. These results were in conformity with Prasannakumar et al., (2009), Ugale et al., (2009), Pradhan et al., (2014), Shashidhar et al., (2000), Sharmili and Manoharan (2018)

\section{Economics}

The growing of little millet + niger in $4: 1$ proportion being at par with treatment $\mathrm{T}_{1}$ i.e. sole little millet registered significantly higher value of gross monetary returns (Rs.45156/-) than all other intercropping systems under pooled mean basis. As regards net monetary returns, three year pooled data revealed that maximum value of Rs.12298/- was noticed with intercropping of little millet + niger in $4: 1\left(\mathrm{~T}_{4}\right)$.

Table.1 Pooled mean growth and yield attributing characters of little millet as influenced by different treatments

\begin{tabular}{|c|c|c|c|}
\hline Treatment & $\begin{array}{l}\text { Plant height } \\
\text { (cm) }\end{array}$ & $\begin{array}{l}\text { Number of } \\
\text { tillers plant }^{-1}\end{array}$ & $\begin{array}{c}\text { Length of } \\
\text { panicle }(\mathrm{cm})\end{array}$ \\
\hline $\begin{array}{l}\mathbf{T}_{1} \text { - Sole Little millet } \\
\mathbf{T}_{2} \text { - Sole Niger }\end{array}$ & $\begin{array}{c}94.9 \\
-\end{array}$ & $\begin{array}{c}8.8 \\
-\end{array}$ & $\begin{array}{c}28.6 \\
-\end{array}$ \\
\hline$T_{3}-$ Little millet + Niger $(2: 1)$ & 86.1 & 8.0 & 20.9 \\
\hline$T_{4}-$ Little millet + Niger $(4: 1)$ & 86.3 & 7.5 & 25.0 \\
\hline$T_{5}-$ Little millet + Niger $(6: 1)$ & 90.5 & 7.5 & 26.7 \\
\hline $\mathbf{T}_{6}-$ Little millet + Niger $(2: 2)$ & 86.7 & 7.9 & 22.5 \\
\hline$T_{7}-$ Little millet + Niger $(4: 2)$ & 88.1 & 8.3 & 25.0 \\
\hline$T_{8}-$ Little millet + Niger $(6: 2)$ & 89.9 & 8.5 & 27.4 \\
\hline $\mathbf{T}_{9}$ - Farmers practice & 79.8 & 5.9 & 18.8 \\
\hline S.E. \pm & 0.96 & 0.09 & 1.18 \\
\hline C.D. 0.05 & 2.92 & 0.27 & 3.57 \\
\hline
\end{tabular}


Table.2 Pooled mean grain and straw yield ( $\mathrm{q} / \mathrm{ha}$ ) of little millet as influenced by different treatments

\begin{tabular}{|c|c|c|c|c|c|c|c|c|}
\hline \multirow[t]{2}{*}{ Treatment } & \multicolumn{3}{|c|}{$\begin{array}{l}\text { Grain yield } \\
\left(\mathbf{q} \mathbf{h a}^{-1}\right)\end{array}$} & \multirow[t]{2}{*}{$\begin{array}{l}\text { Pooled } \\
\text { Mean }\end{array}$} & \multicolumn{3}{|c|}{$\begin{array}{c}\text { Straw yield } \\
\left(\mathbf{q} \mathbf{h a}^{-1}\right)\end{array}$} & \multirow[t]{2}{*}{$\begin{array}{l}\text { Pooled } \\
\text { Mean }\end{array}$} \\
\hline & 2016 & 2017 & 2018 & & 2016 & 2017 & 2018 & \\
\hline $\mathbf{T}_{1}$ - Sole Little millet & 10.80 & 9.76 & 8.77 & 9.78 & 14.04 & 12.99 & 12.46 & 13.16 \\
\hline$T_{2}-$ Sole Niger & 3.06 & 3.02 & 2.66 & 2.91 & 3.60 & 3.50 & 3.40 & 3.50 \\
\hline $\begin{array}{c}T_{3}-\text { Little millet }+ \text { Niger } \\
(2: 1)\end{array}$ & 5.22 & 4.42 & 3.92 & 4.52 & 9.68 & 9.38 & 7.90 & 8.99 \\
\hline$T_{4}-$ Little millet + Niger & 8.07 & 7.86 & 7.41 & 7.78 & 7.40 & 6.74 & 9.75 & 7.96 \\
\hline$T_{5}-$ Little millet + Niger & 8.20 & 7.71 & 7.12 & 7.68 & 10.67 & 8.89 & 9.39 & 9.65 \\
\hline $\begin{array}{c}T_{6}-\text { Little millet }+ \text { Niger } \\
(2: 2)\end{array}$ & 5.63 & 5.72 & 5.23 & 5.53 & 7.31 & 7.03 & 7.03 & 7.13 \\
\hline $\begin{array}{c}T_{7}-\text { Little millet }+ \text { Niger } \\
(4: 2)\end{array}$ & 7.83 & 7.43 & 6.93 & 7.40 & 10.18 & 9.39 & 8.65 & 9.41 \\
\hline $\begin{array}{c}T_{8}-\text { Little millet }+ \text { Niger } \\
(6: 2)\end{array}$ & 7.74 & 7.13 & 6.88 & 7.25 & 10.06 & 9.19 & 8.47 & 9.24 \\
\hline$T_{9}$ - Farmers practice & 6.75 & 6.72 & 5.98 & 6.48 & 8.79 & 7.61 & 7.36 & 7.92 \\
\hline S.E. \pm & 0.44 & 0.58 & 0.63 & 0.16 & 0.54 & 0.62 & 0.67 & 0.47 \\
\hline C.D. 0.05 & 1.35 & 1.76 & 1.90 & 0.47 & 1.64 & 1.87 & 2.03 & 1.43 \\
\hline
\end{tabular}

Table.3 Pooled mean intercrop yield (q/ha) and LER of little millet as influenced by different treatments

\begin{tabular}{|c|c|c|c|c|c|c|c|c|}
\hline \multirow[t]{2}{*}{ Treatment } & \multicolumn{3}{|c|}{$\begin{array}{c}\text { Intercrop yield } \\
\left(\mathbf{q} \mathbf{h a}^{-1}\right)\end{array}$} & \multirow[t]{2}{*}{$\begin{array}{l}\text { Pooled } \\
\text { Mean }\end{array}$} & \multicolumn{3}{|c|}{ LER } & \multirow[t]{2}{*}{$\begin{array}{l}\text { Pooled } \\
\text { Mean }\end{array}$} \\
\hline & 2016 & 2017 & 2018 & & 2016 & 2017 & 2018 & \\
\hline $\begin{array}{c}\mathbf{T}_{1} \text { - Sole Little millet } \\
\mathbf{T}_{2} \text { - Sole Niger }\end{array}$ & 3.06 & 3.02 & 2.66 & 2.91 & $\begin{array}{l}1.00 \\
1.00\end{array}$ & $\begin{array}{l}1.00 \\
1.00\end{array}$ & $\begin{array}{l}1.00 \\
1.00\end{array}$ & $\begin{array}{l}1.00 \\
1.00\end{array}$ \\
\hline$T_{3}$ - Little millet + Niger & 1.04 & 1.16 & 1.23 & 1.14 & 1.03 & 0.85 & 0.92 & 0.93 \\
\hline $\begin{array}{c}T_{4}-\text { Little millet }+ \text { Niger } \\
(4: 1)\end{array}$ & 1.42 & 1.56 & 1.56 & 1.51 & 1.35 & 1.34 & 1.45 & 1.38 \\
\hline $\mathbf{T}_{5}-$ Little millet + Niger & 0.45 & 0.53 & 0.56 & 0.51 & 0.91 & 0.98 & 1.04 & 0.98 \\
\hline $\begin{array}{c}T_{6} \text {-Little millet }+ \text { Niger } \\
(2: 2)\end{array}$ & 0.97 & 1.06 & 1.26 & 1.10 & 1.15 & 0.94 & 1.08 & 1.06 \\
\hline $\begin{array}{c}T_{7}-\text { Little millet }+ \text { Niger } \\
(4: 2)\end{array}$ & 0.94 & 0.97 & 0.97 & 0.96 & 1.06 & 1.11 & 1.18 & 1.12 \\
\hline $\begin{array}{c}T_{8} \text { - Little millet }+ \text { Niger } \\
(6: 2)\end{array}$ & 0.78 & 0.85 & 0.85 & 0.83 & 0.98 & 1.01 & 1.12 & 1.04 \\
\hline $\mathrm{T}_{9}$ - Farmers practice & 0.46 & 0.52 & 0.53 & 0.50 & 0.78 & 0.87 & 0.88 & 0.84 \\
\hline S.E. \pm & -- & -- & -- & -- & -- & -- & -- & -- \\
\hline C.D. 0.05 & -- & -- & -- & -- & -- & -- & -- & -- \\
\hline
\end{tabular}


Table.4 Pooled gross monetary returns $\left(\mathrm{Rs} \mathrm{ha}^{-1}\right)$ and LMGEY (q/ha) of little millet as influenced by different treatments

\begin{tabular}{|c|c|c|c|c|c|c|c|c|}
\hline \multirow[t]{2}{*}{ Treatment } & \multicolumn{3}{|c|}{$\begin{array}{c}\text { Gross monetary returns } \\
\left(\text { Rs ha }^{-1}\right)\end{array}$} & \multirow[t]{2}{*}{$\begin{array}{l}\text { Pooled } \\
\text { Mean }\end{array}$} & \multicolumn{3}{|c|}{$\begin{array}{l}\text { LMGEY } \\
\left(\mathbf{q} \text { ha }^{-1}\right)\end{array}$} & \multirow[t]{2}{*}{$\begin{array}{l}\text { Pooled } \\
\text { Mean }\end{array}$} \\
\hline & 2016 & 2017 & 2018 & & 2016 & 2017 & 2018 & \\
\hline$T_{1}$ - Sole Little millet & 44599 & 45495 & 40714 & 43603 & 14.00 & 11.31 & 9.05 & 10.50 \\
\hline$T_{2}$ - Sole Niger & 21803 & 22414 & 19933 & 21383 & 3.11 & 6.18 & 2.66 & 3.98 \\
\hline$T_{3}$ - Little millet + Niger $(2: 1)$ & 28855 & 28927 & 27661 & 28481 & 7.21 & 7.23 & 6.15 & 6.86 \\
\hline$T_{4}-$ Little millet + Niger $(4: 1)$ & 43249 & 46151 & 46067 & 45156 & 10.81 & 11.75 & 10.24 & 10.93 \\
\hline$T_{5}-$ Little millet + Niger $(6: 1)$ & 37028 & 39251 & 37181 & 37820 & 9.26 & 9.82 & 8.26 & 9.11 \\
\hline$T_{6}$-Little millet + Niger $(2: 2)$ & 30005 & 33889 & 33698 & 32581 & 7.50 & 8.47 & 7.49 & 7.83 \\
\hline$T_{7}-$ Little millet + Niger $(4: 2)$ & 38929 & 41193 & 39383 & 39835 & 9.73 & 10.30 & 8.75 & 9.59 \\
\hline$T_{8}-$ Little millet + Niger $(6: 2)$ & 37463 & 38917 & 38158 & 38179 & 9.37 & 9.73 & 8.48 & 9.19 \\
\hline$T_{9}$ - Farmers practice & 31095 & 34633 & 31609 & 32446 & 7.77 & 8.66 & 7.02 & 7.82 \\
\hline S.E. \pm & 1944 & 1944 & 2992 & 710 & -- & 0.61 & 0.66 & 0.31 \\
\hline C.D. 0.05 & 5826 & 5826 & 8967 & 2127 & -- & 1.84 & 1.97 & 0.93 \\
\hline
\end{tabular}

Table.5 Pooled net monetary returns $\left(\mathrm{Rs} \mathrm{ha}^{-1}\right)$ and $\mathrm{B}$ : $\mathrm{C}$ ratio as influenced by different treatments

\begin{tabular}{|c|c|c|c|c|c|c|c|c|}
\hline \multirow[t]{2}{*}{ Treatment } & \multicolumn{3}{|c|}{$\begin{array}{c}\text { Net monetary returns } \\
\left(\mathrm{Rs} \mathrm{ha}^{-1}\right)\end{array}$} & \multirow[t]{2}{*}{$\begin{array}{c}\text { Pooled } \\
\text { Mean }\end{array}$} & \multicolumn{3}{|c|}{ B : C Ratio } & \multirow[t]{2}{*}{$\begin{array}{l}\text { Pooled } \\
\text { Mean }\end{array}$} \\
\hline & 2016 & 2017 & 2018 & & 2016 & 2017 & 2018 & \\
\hline $\mathbf{T}_{1}$ - Sole Little millet & 11284 & 11910 & 7935 & 10376 & 1.34 & 1.36 & 1.24 & 1.31 \\
\hline $\mathbf{T}_{2}$ - Sole Niger & -11512 & -10901 & -12847 & -11753 & 0.65 & 0.67 & 0.61 & 0.64 \\
\hline$T_{3}-$ Little millet + Niger $(2: 1)$ & -4460 & -4388 & -5119 & -4656 & 0.87 & 0.87 & 0.84 & 0.86 \\
\hline$T_{4}-$ Little millet + Niger $(4: 1)$ & 9935 & 13671 & 13287 & 12298 & 1.30 & 1.41 & 1.41 & 1.37 \\
\hline$T_{5}-$ Little millet + Niger $(6: 1)$ & 3713 & 5969 & 4401 & 4694 & 1.11 & 1.18 & 1.13 & 1.14 \\
\hline$T_{6}$-Little millet + Niger $(2: 2)$ & -3310 & 574 & 918 & -606 & 0.90 & 1.02 & 1.03 & 0.98 \\
\hline$T_{7}-$ Little millet + Niger $(4: 2)$ & 5614 & 7878 & 6603 & 6698 & 1.17 & 1.24 & 1.20 & 1.20 \\
\hline$T_{8}-$ Little millet + Niger $(6: 2)$ & 4149 & 5602 & 5377 & 5043 & 1.12 & 1.17 & 1.16 & 1.15 \\
\hline$T_{9}$ - Farmers practice & 715 & 4254 & 1679 & 2216 & 1.02 & 1.14 & 1.06 & 1.07 \\
\hline S.E. \pm & -- & -- & -- & -- & -- & -- & -- & -- \\
\hline C.D. 0.05 & -- & -- & -- & -- & -- & -- & -- & -- \\
\hline
\end{tabular}

The same treatment i.e. $\mathrm{T}_{4}$ also showed its superiority and recorded highest benefit cost ratio value of 1.37 as compared all other intercropping treatments.

The increased gross and net returns and B: C ratio in intercropping system was mainly due to higher little millet rain yield. These results are in conformity with findings of by
Shashidhar et al., (2000), Patil et al., (2010). Ugale et al., (2009), Sharmili and Manoharan (2018). From these three year pooled data, it could be concluded that growing of little millet + niger in 4:1 row ratio is the most efficient and profitable intercropping system on shallow entisols of Sub-montane zone of Maharashtra. 


\section{Acknowledgments}

Authors are thankful to authorities of All India Coordinated Small Improvement Project, Zonal Agricultural Research Station, Shenda Park, Kolhapur for conduct of research, support and encouragement during experimentation.

\section{References}

Mangunath M. G. and Salankikop 2017. Growth and yield of soybean and millets in intercropping systems. $S . R$. J. Farm Sci., 30(3): 349-353.

Panse V. G. and Sukhatme P. V. 1985. Statistical methods for agricultural workers. ICAR, New Delhi, pp. 145148.

Patil N.B, Halikatti S.I., Sujay Y.H., Prasanna Kumar B.H, Toagi Sanjay C. and Pushpa V. 2010. Intercrop association of pigeon pea (Cajanus cajan) with little millet (Panicum sumatrence L.) Internat. J. agric. Sci., $6(2): 602-604$

Pradhan Adikant, Thakur A, Sao, A and Patel D.P. 2014. Biological efficiency of intercropping in finger millet (Eleusine coracan L.) under raifed condition. Int. J. Curr. Microb. Appl. Sci., 3 (1) :719-723.

Prasannakumar, B.H., Hallikatti, S.I.
Ninganur, B.T. 2009. Sustainable intercrop association of pigeonpea (Cajanas cajan) in little millet. Karnataka I. Agric. Sci. 22(4): 887-888. Sharmili, K. and Manoharan, S. 2018. Studies on Intercropping in Rainfed Little Millet (Panicum sumatrense). Int . J. Curr. Microbiol. App. Sci. 7(02): 323327.

Shashidhar, G.B., Basavaraj za, R. and Nadagouda, B. 2000. Studies on pigeonpea intercropping systems in small millets under shallow red soils. Karnataka J. agric. Sci., 13 (1): 7-10.

Tripathi, A.K. and Kushwaha, H.S. 2013. Performance of pearl millet (Pennisetum glaucum) intercropped with pigeonpea (Cajanus cajan) under varying fertility levels in the rainfed environment of Bundelkhand region. Ann. Agric. Res. New Series. 34: 36-43.

Uagle, A.N. Sawant A.C. and Chavan P.G. 2009. Effect of intercropping of niger in kharif proso millet (Panicum miliaceum L.) with organic and inorganic sources of nutrients on yield, economics and intercropping indices. Internat. J. agric. Sci. 5 (2) 346-348.

Willey, R.W. 1979. Intercropping its importance and research needs Part 1 competition and yield advantages. Fld. Crops Sci. Abstact, 32 (1) : 1-10.

\section{How to cite this article:}

Nigade. R. D., R. L. Bhilare and Bagade. S. V. 2020. Sustainable Intercropping Association of Little Millet (Panicum sumatrense L.) in Niger (Guizotia abyssinica Cass). Int.J.Curr.Microbiol.App.Sci. 9(06): 660-665. doi: https://doi.org/10.20546/ijcmas.2020.906.084 